УДК 616.314-72

DOI

\title{
МЕЖДИСЦИПЛИНАРНЫЙ ПОДХОД В ЛЕЧЕНИИ ОРОФАЦИАЛЬНОЙ БОЛИ И ПАТОЛОГИИ ВИСОЧНО-НИЖНЕЧЕЛЮСТНОГО СУСТАВА У ПАЦИЕНТОВ С ПОЛНЫМ ИЛИ ЧАСТИЧНЫМ ОТСУТСТВИЕМ ЗУБОВ (ОБЗОР ЛИТЕРАТУРЫ)
}

\section{AN INTERDISCIPLINARY APPROACH IN THE TREATMENT OF OROFACIAL PAIN AND PATHOLOGY OF THE TEMPOROMANDIBULAR JOINT IN PATIENTS WITH COMPLETE OR PARTIAL ADENTIA (REVIEW OF LITERATURE)}

\author{
Ф.К. Дзалаева ${ }^{1}$, С.О. Чикунов ${ }^{1,2}$, А.С. Утюж ${ }^{1}$, М.В. Михайлова ${ }^{1}$, М.К. Будунова ${ }^{1}$ \\ F.K. Dzalaeva ${ }^{1}$, S.O. Chikunov ${ }^{1,2}$, A.S. Utyuzh ${ }^{1}$, M.V. Mikhailova ${ }^{1}$, M.K. Budunova ${ }^{1}$ \\ ${ }^{1}$ Кафедра ортопедической стоматологии, Первый Московский государственный медицинский \\ университет имени И.М. Сеченова, Россия, 121614, г. Москва, ул. Большая Пироговская, 19/1 \\ Department of prosthodontics, First Moscow State Medical University, \\ 19/1 Bolshaya Pirogovskaya St, Moscow, 121614, Russia
}

${ }^{2}$ Российский университет дружбы народов, Россия, г. Москва, 117198, ул. Миклухо-Маклая, 6 Peoples friendship university (RUDN), 6 Miklukho-Maklaya St, Moscow, 117198, Russia

E-mail: dzalayevaf1624@bk.ru

\begin{abstract}
Аннотация
В последние десятилетия появляется все больше сообщений о взаимосвязи и важности оценки в ходе обследования пациентов с адентией, нуждающихся в стоматологическом лечении и ортодонтической реабилитации таких патологических проявлений, как орофациальная боль, височно-нижнечелюстная патология и изменения окклюзия. Цель работы - обоснование необходимости применения междисциплинарного подхода к лечению пациентов с адентией на основании анализа литературных данных о взаимосвязи клинических проявлений орофациальной боли, окклюзии и патологии височно-нижнечелюстного сустава у пациентов с полным и частичным отсутствием зубов. Описаны классификация, этиология и проявления орофациального болевого синдрома. Указано, что в большинстве случаев при планировании комплексной ортодонтической реабилитации необходимо обеспечивать создание определенных взаимно защитных окклюзионных схем путем коррекции положения челюстей, в частности за счет обеспечения приемлемых окклюзионных вертикальных размеров. Необходимость использования междисциплинарного подхода к проведению стоматологического лечения данного контингента пациентов предусматривает в первую очередь, что пациентам с орофациальной болью необходимо проведение тщательного комплексного обследования. В связи с этим обязательной является оценка состояния височно-нижнечелюстного сустава с помощью комплекса инструментальных и лабораторных методов диагностики. Отмечена важность пальпации шейных мышц, этот элемент должен стать обязательной частью стандартного обследования данной категории пациентов. Наиболее эффективным в решении проблемы орофациальной боли и патологии височнонижнечелюстного сустава является сочетание стоматологического, ортопедического и остеопатического лечения. При этом в ряде случаев первостепенное значение имеют методы психодиагностики и психокоррекции. Независимо от цели проводимых лечебнореабилитационных мероприятий специалисты должны максимально прилагать усилия для минимизации влияний установленных конструкций на состояние центральной нервной системы пациентов. Сделано заключение о том, что в рамках комплексного междисциплинарного подхода к диагностике и лечению пациентов с адентией необходимо использование методов, подтвержденных соответствующей доказательной базой, с целью верификации преобладания болевых нейромышечных или окклюзионно-артикулярных синдромов, а также оценки их системного влияния на биомеханические взаимоотношения всех элементов челюстно-лицевой системы.
\end{abstract}




\begin{abstract}
In recent decades, there are more reports about the relationship and importance of evaluating pathological manifestations such as orofacial pain, temporomandibular pathology and occlusion changes during the examination of patients with adentia who need dental treatment and orthodontic rehabilitation. Aim of the work is to justify the need for an interdisciplinary approach to the treatment of patients with adentia based on literature data analysis on the relationship of orofacial pain, occlusion and temporomandibular joint pathology in patients with complete and partial adentia. It described the classification, etiology and manifestations of orofacial pain syndrome. It is indicated that in most cases when planning a complex orthodontic rehabilitation it is necessary to ensure the protective occlusal schemes by correcting the position of the jaws, in particular by ensuring acceptable occlusal vertical dimensions. The first need to use an interdisciplinary approach to the dental treatment of this patient population provides that patients with orofacial pain need a thorough comprehensive examination. In this regard, it is mandatory to assess the condition of the temporomandibular joint using a complex of instrumental and laboratory diagnostic methods. The importance of palpation of the cervical muscles was noted, this element should become an obligatory part of the standard examination. The most effective in orofacial pain and temporomandibular joint pathology a combination of dental, orthopedic, and osteopathic treatment. Moreover, in some cases, methods of psychodiagnostics and psychocorrection are of paramount importance. Regardless of the purpose of the treatment and rehabilitation measures, specialists should make maximum efforts to minimize the effects of established structures on the state of the patient central nervous system. It is concluded that, as part of a comprehensive interdisciplinary approach to the diagnosis and treatment of patients with adentia, it is necessary to use methods confirmed by the corresponding evidence base in order to verify the prevalence of painful neuromuscular or occlusal-articular syndromes, as well as assess their systemic effect on the biomechanical relationships of all elements maxillofacial system.
\end{abstract}

Ключевые слова: адентия, височно-нижнечелюстной сустав, окклюзия, орофациальная боль, междисциплинарный подход.

Keywords: adentia, temporomandibular joint, occlusion, orofacial pain, interdisciplinary approach.

\title{
Введение
}

Орофациальную боль определяют как «боль, локализованную над шеей, в передней околоушной области, ниже линии орбиты, а также боль в полости рта, включая зубную боль, а также боль, обусловленную височно-нижнечелюстной патологией (ВНП)» [Shephard et al., 2013; Ohrbach, Dworkin, 2016; Milosevic, 2017]. В свою очередь ВНП определяют как «условия, способствующие неполной или нарушенной функции височнонижнечелюстного сустава (ВНЧС) и/или жевательных мышц». Окклюзия определяется как «статическая взаимосвязь между резцовыми и/или жевательными поверхностями зубов верхней и нижней челюсти» [Ohrbach, Dworkin, 2016].

В последние десятилетия появляется все больше сообщений о взаимосвязи и важности оценки в ходе обследования пациентов с адентией, нуждающихся в стоматологическом лечении и ортодонтической реабилитации таких патологических проявлений, как орофациальная боль, ВНП и изменения окклюзии. Показана роль нарушений состояния ВНЧС, учет этих патологических проявлений является крайне важным при планировании лечебно-реабилитационных мероприятий у данной категории пациентов. По данным разных авторов, от 27 до 76 \% пациентов, обращающихся к стоматологам, предъявляют жалобы на нарушение функции ВНЧС [Yau et al., 2014; Milosevic, 2017].

Виды адентии, анатомические и функциональные особенности зубочелюстной системы, возникающие после утраты зубов, обусловливают выбор и использование методов лечебно-реабилитационных мероприятий с применением протезов, различных по форме, размерам и конструкции. Как показывает анализ данных литературы, эффективность лечения определяется не столько видом и торговой маркой используемых изделий, сколько дифференцированным подходом - рациональным выбором конструкции протеза, правильным его изготовлением и фиксацией [Shephard et al., 2013; Yau et al., 2014; McSwiney et al., 2017]. При этом независимо от цели лечения специалисты должны прилагать усилия 
для минимизации влияний установленных конструкций на состояние центральной нервной системы (ЦНС) пациентов. Такие влияния могут быть вызваны, в частности, нарушениями окклюзионных соотношений, способствующих проявлениям орофациальной боли и височно-нижнечелюстной патологии [Durham et al., 2015; Baad-Hansen, Benoliel, 2017]. Однако работы, посвященные различным аспектам этих патологических проявлений, особенностям их диагностики и лечения в доступной литературе немногочисленны.

Цель работы - обоснование необходимости применения междисциплинарного подхода к лечению пациентов с адентией на основании анализа литературных данных о взаимосвязи клинических проявлений орофациальной боли, окклюзии и патологии височно-нижнечелюстного сустава у пациентов с полным и частичным отсутствием зубов.

Острые и хронические проявления орофациального болевого синдрома подразделяют на 3 основные категории: соматические, нейрогенные и психогенные [Durham et al., 2015; Baad-Hansen, Benoliel, 2017; Ghurye, McMillan, 2017]. Острая боль в челюстнолицевой области часто проявляется, например, при таких состояниях, как афтозные язвы и пульпит, относительно легко купируется. В то же время хроническая боль имеет тенденцию к рефрактерности, что обусловливает сложность лечения проявлений этого синдрома. Соматическая боль (в области мягких или твердых тканей) характеризуется, как правило, как тупая, болезненная, пульсирующая, термически чувствительная.

\section{Результаты и их обсуждение}

Нейропатическая боль (часто в результате повреждения нерва) описывается как жжение или колющая боль. Психогенная боль (особенно соматоформная) не вызвана соматической патологией, но, как правило, связана с эмоциональными расстройствами, изменениями настроения и когнитивными нарушениями. Часто у пациентов с челюстнолицевой патологией отмечаются проявления одновременно двух или трех этих основных вариантов орофациальной боли [Merrill, Goodman, 2016; Fishbain et al., 2017].

Афферентная сенсорная информация из лицевой области поступает в ЦНС через тройничный (V) черепно-мозговой нерв [Shankland, 2000]. Информация, поступающая по афферентным импульсам, сигнализирует о термических, механических, механоцептивных или ноцицептивных стимулах, воздействующих на организм. Эта информация поступает в область хвостатого ядра и распространяется по нейронам первого порядка лицевого (VII), глоссофарингеального (IX), блуждающего (X), гипоглоссального (XI) и первых трех шейных нервов (от С1 до С3) [Shankland, 2000].

Конвергенция черепно-мозговых и шейных нервов является одним из условий возникновения рассматриваемых видов болевых проявлений, в связи с чем место проявления и источник боли могут различаться. Нейроны второго порядка передают афферентную информацию в таламус и третичные нейроны, таким образом завершая передачу информации в кору головного мозга. После поступления в кору ноцицептивной информации человек испытывает боль. К настоящему времени установлено, что в ЦНС существует множество путей модуляции ноцицептивной информации как в процессе ее поступления по восходящему пути (в ретикулярную формацию), так и при нисходящей передаче (серое ядро моста) [Sessle et al., 1986; Saito et al., 2017]. В настоящее время предложены нейроматрицы для описания сложных взаимодействий в ЦНС, которые реализуются за счет различных нейротрансмиттеров (таких как эндорфины, дофамин, серотонин), и в конечном итоге приводят к клиническим проявлениям патологии, в данном случае - боли [Melzack, 2001].

Нейропластичность ЦНС человека способствует развитию ее сенсибилизации, усилению либо ослаблению регуляторных воздействий, что, в частности, может реализовываться за счет активации глиальных клеток [Ohkubo et al., 2013; Yu, Abbott, 2016]. Ноцицептивные сигналы могут поступать в ЦНС из пульпы зуба (синдром трещины зуба, возбуждение одонтобласта, патология пульпы), что является примером центральных нейро- 
пластических изменений с сопутствующими физиологическими и клиническими проявлениями [Melzack, 2001; Ohkubo et al., 2013]. Центральная сенсибилизация может также стимулировать эфферентное выделение нейровоспалительных медиаторов и представляет собой общий патофизиологический механизм, лежащий в основе развития ряда хронических болевых состояний, таких как ТМП, атипичная одонталгия, синдром хронической усталости, синдром регионарной боли, дисменорея, синдром раздраженного кишечника, постоянная идиопатическая орофациальная и головная боль [Robinson et al., 2016].

K настоящему времени установлено наличие большого количества различных патологических взаимовлияний и патогенетических механизмов в ЦНС вследствие ноцицептивных воздействий из области головы и шеи, что обусловливает достаточно часто наблюдающиеся неверные интерпретации специалистами клинических проявлений патологии и ошибочные диагнозы [Gauer, Semidey, 2015]. Следовательно, стоматологам необходимо иметь четкие представления о патогенезе орофациальной боли, особенностях патологических проявлений нарушений в ВНЧС и необходимости учета состояния челюстно-лицевой области при проведении стоматологического и ортодонтического лечения. Представляется оптимальным использование комплексного мультидисциплинарного подхода к лечению этих проявлений.

Важнейшую роль в развитии заболеваний ВНЧС играют аномалии прикуса, частота выявления которых составляет от 34 до $87 \%$ в структуре этой патологии. Большинство авторов полагают, что развитие аномалий соотношения зубов обусловлено в меньшей степени морфологическими особенностями элементов сустава, в большей степени - изменениями внутрисуставных взаимоотношений [Yau et al., 2014; Baad-Hansen, Benoliel, 2017, Kretschmer et al., 2019; Tkachenko et al., 2019].

Окклюзионно-обусловленные изменения суставных поверхностей и капсулы ВНЧС, как правило, усугубляют возрастные изменения, что в свою очередь обусловливает возникновение анатомических предпосылок развития его дисфункции [Saito et al., 2017; Tkachenko et al., 2019]. Показано, что хронический стресс при патологии ВНЧС проявляется в виде парафункций и бруксизма, что способствует появлению чувства «усталости» жевательных мышц, болям при жевании и их спазму. Продемонстрирована также роль психогенных факторов в развитии заболевания [Baad-Hansen, Benoliel, 2017; Kretschmer et al., 2019].

До последнего времени окклюзия рассматривалась как статическая взаимосвязь окклюзирующих поверхностей челюсти и зуба (естественных или протезов), а также имплантата или других ортопедических конструкций [Ferro et al., 2017]. Однако к настоящему времени показано, что состояние окклюзии является в большей степени не статичным, а динамичным. В связи с этим важным представляется информирование и напоминание пациентам о необходимости размыкать зубы во время бодрствования [Racich, 2015]. Bо время сна активные контакты зубов, обусловленные их сжиманием и/или бруксизмом, могут быть достаточно частыми и длительными, в связи с чем следует осуществлять защиту зубов или протезов, например, жесткой акриловой смолой, чтобы минимизировать проявления апноэ во сне и ортопедически неблагоприятные эффекты бруксизма [Manfredini et al., 2017]. У пациентов, которым проводится ортодонтическое, ортопедическое лечение или комплексный уход за полостью рта, необходимо применение защитных окклюзионных схем [Abduo et al., 2013]. В ходе использования подобных реабилитационных подходов обеспечивается приемлемое вертикальное положение мыщелка нижней челюсти в суставной ямке [Moreno-Hay, Okeson, 2015].

При возможности зубной ряд (искусственный или естественный) располагается сагиттально и коронарно, что физиологически обосновано [Porwal, Sasaki, 2013]. Tакое анатомическое положение зубов позволяет обеспечить оптимальную жевательную эффективность [Sheridan et al., 2016; Mousa et al., 2017]. В соответствии с этим изготавливаются и протезы на иплантатах, на которых для обеспечения окклюзии применяется рельефная 
прокладка, осуществляется минимизация кантилевера или шинирование [Sheridan et al., 2016].

В большинстве случаев при планировании комплексной ортодонтической реабилитации необходимо обеспечивать создание определенных взаимно защитных окклюзионных схем путем коррекции положения челюстей, в частности за счет обеспечения приемлемых окклюзионных вертикальных размеров, минимизируя вертикальное перекрытие бруксеров и, соответственно, снижение экскурсионных усилий.

Необходимость использования междисциплинарного подхода к проведению стоматологического лечения данного контингента пациентов предусматривает в первую очередь, что пациентам с орофациальной болью необходимо проведение тщательного комплексного обследования [Dolce et al., 2014]. В связи с этим необходимой является оценка состояния ВНЧС с помощью методов конусной лучевой компьютерной томографии, ультразвуковой допплерографии, магнитно-резонансной томографии. При этом следует учитывать, что, несмотря на то, что эти методы обладают хорошей чувствительностью, в то же время их специфичность является относительно низкой в отношении долгосрочного прогноза развития височно-нижнечелюстной патологии [Bakke et al., 2014].

Для оценки проявлений ВНП предлагается применение и других методов - сканирование костей, определение С-реактивного белка, интерлейкина-6, ревматоидного фактора, антинуклеарных антител. Также предлагается проведение оценки качества сна и выраженности проявлений синдрома обструктивного апноэ сна с помощью соответствующих опросников [Chung et al., 2012]. Следует также осуществлять оценку локальной мышечной болезненности, для определения которой используются миофасциальные триггерные точки, а также выраженность центрально-опосредованного миозита, хотя такие методы, как электромиография, характеризуются относительно низкой специфичностью [Al-Salleh et al., 2012].

Лечение орофациальной боли проводится с использованием ряда применяемых в настоящее время стандартных и альтернативных методов, к которым относятся акупунктура, гомеопатия, натуропатия, остеопатия, физиотерапия и массаж. При этом также используются методы психотерапии и традиционной китайской медицины [Greene, Obrez, 2015].

Специалистам следует обеспечивать благоприятную атмосферу проводимого стоматологического лечения, в ходе которого учитывать диагноз и особенности проводимых лечебно-реабилитационных мероприятий (терапия острой боли, устранение аберрантных ноцицептивных воздействий на ЦНС, источниками которых могут быть изменения окклюзии зубов, наличие ортопедических твердых акриловых смол, специальных стентов, препаратов для местного применения).

Комплексное ортодонтическое, ортопедическое и восстановительное лечение должно рассматриваться как вторичное по отношению к адекватному лечению наблюдаемых проявлений дискомфорта пациента и нарушений функционирования челюстнолицевой системы и организма в целом. В стоматологии лечение миофасциального болевого синдрома достаточно часто проводится с использованием спреев и растираний, реже используются инъекции анестетиков. Медикаментозное лечение, включая использование превентивных методов анальгезии, следует назначать на основании результатов консультаций всех специалистов [Vadivelu et al., 2014]. Совместно с другими специалистами пациентам с орофациальным болевым синдромом и дисфункцией ВНЧС назначают седативные препараты, антидепрессанты, миорелаксанты, антидепрессанты. Применение этих лекарственных средств позволяет снять чувство эмоционального дискомфорта, страха, а также снизить выраженность спазма жевательной мускулатуры и болевого синдрома.

В ряде исследований в устранении болевого синдрома и нормализации функционального состояния жевательных мышц продемонстрирована высокая эффективность магнитолазерной терапии. Анальгезируюшее воздействие лазерного излучения купирует у 
пациентов чувство эмоционального напряжения, тревоги, что также положительно сказывается на процессе лечения [Yau et al., 2014; Baad-Hansen, Benoliel, 2017].

Влияние на дисфункцию височно-нижнечелюстного сустава оказывает позитивное влияние выполнения ортогнатических вмешательств [Kretschmer et al., 2019]. В ряде исследований подтверждена эффективность применения при лечении болевого синдрома с окклюзионными нарушениями в ВНЧС сплинт-терапии в комплексе с ортопедическими и физиотерапевтическими методами. Показано, что окклюзионные шины изменяют характер смыкания зубов, воздействуют на пародонт, жевательные мышцы и ВНЧС [Hu, Dong, 2019].

В исследовании Э.Р. Ордоковой [2018] применение сплинт-терапии способствовало снижению тонуса жевательных мышц у 81,8 \% пациентов, болевой чувствительности у 85,7\%. Автор отмечает наличие положительного результата через 1-1,5 месяца от момента установки репозиционных шин. При этом боли и щелчки в ВПЧС прекратились у 83,3 \% исследуемых. Проведение кинезитерапии позволило через 7 дней добиться купирования болевого синдрома у всех пациентов [Ордокова, 2018]. Другие исследователи также подчеркивают положительную роль кинезитерапии при лечении данной категории пациентов, поскольку применение такого подхода к лечению, как полагают, позволило значительно уменьшить выраженность болевых симптомов [Baad-Hansen, Benoliel, 2017; Иорданишвили, Сериков, 2018].

В отдельных работах продемонстрирована также эффективность остеопатической терапии в комплексном лечении пациентов с дисфункцией ВНЧС [Силаев и др., 2014].

Важным элементом комплексного лечения миофациального синдрома (МФС) являются методы ортодонтического лечения, целью которых является устранение нарушений прикуса. В то же время у специалистов не выработано единого мнения относительно прогноза развития МФС в процессе проведения ортодонтической терапии.

Tecco S. et al. [2012] предприняли попытку проанализировать особенности проявлений МФС в процессе ортодонтического лечения пациентов. Был проведен анализ данных 91 пациента европеоидной расы, которым осуществлялось ортодонтическое лечение различных нарушений прикуса [Tecco et al., 2012].

МФС был первоначально диагностирован у 37 пациентов. Из них 30 пациентам (основная группа) проводилось ортодонтическое лечение, после определения класса коррекции и выравнивания зубов была выполнена оценка проявлений МФС. 7 пациентам, которые составили группу контроля, лечение МФС не проводилось. При обследовании пациентов основной группы после проведенного лечения наблюдалось статистически значимое снижение частоты каких-либо проявлений патологии ВНЧС (в частности, щелчков). Отмечалось также существенное снижение выраженности болевых ощущений в области челюстей, ВНЧС, лицевых мышц. При этом авторы отметили существенное улучшение качества жизни этих пациентов [Tecco et al., 2012].

У части пациентов в начале наблюдения наблюдались признаки депрессии, в дальнейшем отмечалось уменьшение проявлений этих симптомов, большинство пациентов сообщили об улучшении своего субъективного самочувствия по результатам их субъективной оценки. При пальпации мышц было обнаружено статистически значимое снижение выраженности болевых ощущений (при оценке по визуально-аналоговой шкале) в области височной мышцы, ключичного и грудинного отдела грудино-ключично-сосцевидной мышцы, жевательных и задних шейных мышц. В основной группе пациентов после проведенного лечения было отмечено значительное уменьшение количества пациентов с проявлениями болевых ощущений в триггерных точках в области височных и жевательных мышц. Аналогичные изменения были выявлены также в отношении пациентов с подобными проявлениями в области $\mathrm{m}$. digastric и грудино-ключично-сосцевидной мышцы [Tecco et al., 2012].

Целью исследования Emodi Perelman A et al. [2015] явилось сравнение распространенности болей в шейных мышцах (БШМ), а также миогенных нарушений в области 
ВНЧС у врачей-стоматологов женского пола, работников ряда высокотехнологичных сфер, а также пациентов, профессионально занятых в других областях деятельности. Авторы изучали взаимосвязи между проявлениями МФС, патологией ВНЧС и бруксизмом, а также предприняли попытку оценки влияния производственного стресса на выраженность МФС и проявления БШМ.

Оценка этих проявлений проводилась на основании клинических проявлений МФС и БШМ, а также по результатам анкетирования, целью которого была оценка выраженности болевых ощущений и стресса. Диагноз бруксизм в период сна был установлен с использованием утвержденных диагностических критериев Американской академии медицины сна (Международная классификация нарушений сна (ICSD-2), 2005, Вестчестер, Иллинойс), диагноз бруксизм в период бодрствования был установлен по результатам проведенного анкетирования [Emodi Perelman et al., 2015].

Было установлено, что величина показателя отношения шансов (ОШ) для пациентов с МФС, у которых одновременно проявлялись признаки БШМ или бруксизма (во время сна и/или бодрствования), варьировала от 2,603 до 3,077. По мнению авторов, полученные данные свидетельствовали о том, что как специалисты-стоматологи, так и работники высокотехнологичных производств подвержены более высокому риску развития патологии ВНЧС и болевых проявлений в области мышц шеи и лица по сравнению с работниками других профессий.

Исследователи подчеркивают, что выявленные в данном исследовании ассоциации вышеописанных клинических проявлений свидетельствуют о важности пальпации шейных мышц, этот элемент должен стать обязательной частью стандартного обследования при подозрении на наличие патологии ВНЧС [Emodi Perelman et al., 2015].

\section{Заключение}

В последние годы отмечаются большие достижения в области развития технологий стоматологической ортодонтической помощи, в том числе в отношении лечения орофациальной боли, височно-нижнечелюстной патологии и нарушений окклюзии в процессе проведения ортодонтического, ортопедического и восстановительного лечения. Междисциплинарная многопрофильная помощь стала практической реальностью в условиях современного интегративного здравоохранения.

Мультифакториальность проблемы, изложенной в данной статье, в значительной степени определяет структуру необходимых диагностических и лечебных мероприятий при планировании стоматологического и ортодонтического лечения и реабилитации пациентов. Мы согласны с мнением ряда авторов, что наиболее эффективным в решении проблемы орофациальной боли и патологии ВНЧС является сочетание стоматологического, ортопедического, и остеопатического лечения. При этом в ряде случаев первостепенное значение имеют методы психодиагностики и психокоррекции. Необходимо взаимодействие врачей разных специальностей с целью комплексной оценки клинической ситуации, разработки оптимального алгоритма диагностики и лечения.

В рамках комплексного междисциплинарного подхода к диагностике необходимо использование диагностических методов исследования с соответствующей доказательной базой с целью верификации преобладания болевых нейромышечных или окклюзионноартикулярных синдромов, а также оценки их системного влияния на биомеханические взаимоотношения всех элементов челюстно-лицевой системы.

\section{Список литературы}

1. Иорданишвили А.К., Сериков А.А. 2018. О необходимости стандартизации подходов к лечению и реабилитации пациентов с патологией височно-нижнечелюстного сустава в учреждениях разной формы собственности. Медицинский вестник Северного Кавказа. 13 (1-1): 59-62. 
2. Ордокова Э.Р. 2018. Диагностика и лечение дисфункции височно-нижнечелюстного сустава у пациентов с аномалиями прикуса. Смоленский медицинский альманах. 2: 55-57.

3. Силаев А.М., Зубова К.Н., Новосельцев С.В. 2014. Остеопатические возможности диагностики и лечения синдрома дисфункции височно-нижнечелюстного сустава. Мануальная терапия. 4: 20-31.

4. Abduo J., Tennant M., McGeachie J. 2013. Lateral occlusion schemes in natural and minimally restored permanent dentition: a systematic review. J Oral Rehabil. 40: 788-802.

5. Al-Salleh M.A., Armijo-Olivo S., Flores-Mir C., Thie N.M. 2012. Electromyography in diagnosing temporomandibular disorders. J Am Dent Assoc. 143: 351-362.

6. Baad-Hansen L., Benoliel R. 2017. Neuropathic orofacial pain: facts and fifiction. Cephalalgia. 37: 670-679.

7. Bakke M., Petersson A., Wiesel M., Svanholt P., Sonnesen L. 2014. Bony dethroughtions revealed by cone beam computed tomography of the temporomandibular joint in subjects without ongoing pain. J Oral Facial Pain Headache. 28: 331-337.

8. $\quad$ Chung F., Subramanyam R., Liao P., Sasaki E., Shapiro C., Sun Y. 2012. High stop bang score indicates a high probability of obstructive sleep apnoea. Br J Anaesth. 108: 768-775.

9. Dolce M.C., Aghazadeh-Sanai N., Mohammed S., Fulmer T.T. 2014. Integrating oral health into the interdisciplinary health sciences curriculum. Dent Clin North Am. 58: 829-843.

10. Durham J., Raphael K.G., Benoliel R., Ceusters W., Michelotti A., Ohrbach R. 2015. Perspectives on next steps in classifification of oro-facial pain - part 2: role of psychosocial factors. J Oral Rehabil. 42: 942-955.

11. Emodi Perelman A., Eli I., Rubin P.F., Greenbaum T., Heiliczer S., Winocur E. 2015. Occupation as a potential contributing factor for temporomandibular disorders, bruxism, and cervical muscle pain: a controlled comparative study. Eur J Oral Sci. 123 (5): 356-361.

12. Ferro K.J., Morgano S.M., Driscoll C.F., Freilich M.A., Guckes A.D., Knoernschild K.L., VcGarry T.J. 2017. The glossary of prosthodontic terms. Ninth edition. J. Prosthet. Dent. 117 (5): 1-105.

13. Fishbain D.A., Pulikal A., Lewis J.E., Gao J. 2017. Chronic pain types differ in their reported prevalence of post-traumatic stress disorder (PTSD) and there is consistent evidence that chronic pain is associated with ptsd: an evidence based structured systematic review. Pain Med. 18: 711-735.

14. Gauer R.L., Semidey M.J. 2015. Diagnosis and treatment of temporomandibular disorders. Am Fam Physician. 91: 378-386.

15. Ghurye S., McMillan R. 2017. Orofacial pain: an update on diagnosis and man agement. Br. Dent. J. 223: 639-647.

16. Greene C.S., Obrez A. 2015. Treating temporomandibular disorders with permanent mandibular repositioning: is it medically necessary? Oral Surg., Oral Med., Oral Pathol., Oral Radiol. 119: 489-498.

17. Hu J.L., Dong Y. 2019. Research progress in occlusal splint therapy for disorders. Zhonghua Kou Qiang Yi Xue Za Zhi. 54 (4): 273-277.

18. Kretschmer W.B., Baciuț G., Baciuţ M., Sader R. 2019. Effect of bimaxillary orthognathic surgery on dysfunction temporomandibular joint: a retrospective study of 500 consecutive cases. Br. J. Oral Maxillofac. Surg. 57 (8): 734-739.

19. Manfredini D., Serra-Negra J., Carboncini F., Lobbezoo F. 2017. Current concepts of bruxism. Int. J. Prosthodont. 30: 437-438.

20. McSwiney T.P., Collins J.M., Bassi G.S., Khan S. 2017. The interdisciplinary management of hypodontia patients in the UK: a national service evaluation. Br. Dent. J. 222 (1): 31-35.

21. Melzack R. 2001. Pain and the neuromatrix in the brain. J. Dent. Educ. 65: 1378-1382.

22. Merrill R.L., Goodman D. 2016. Chronic orofacial pain and behavioral medicine. Oral Maxillofac. Surg. Clin. North. Am. 28: 247-260.

23. Milosevic A. 2017. Abrasion: A Common Dental Problem Revisited. Prim. Dent. J. 6 (1): $32-36$.

24. Moreno-Hay I., Okeson J.P. 2015. Does altering the occlusal vertical dimension produce temporomandibular disorders? A literature review. J. Oral Rehabil. 42: 875-882.

25. Mousa M.A., Patil S., Lynch E. 2017. Masticatory effificiency and muscular activity in removable partial dental prostheses with different cusp angles. J. Prosthet. Dent. 117: 55-60.

26. Ohkubo C., Morokuma M., Yoneyama Y., Matsuda R., Lee J.S. 2013. Interactions between occlusion and human brain function activities. J. Oral Rehabil. 40: 119-129. 
27. Ohrbach R., Dworkin S.F. 2016. The evolution of TMD diagnosis: past, present, future. J. Dent. Res. 95: 1093-1101.

28. Porwal A., Sasaki K. 2013. Current status of the neutral zone: a literature review. J. Prosthet. Dent. 109: 129-134.

29. Racich M.J. 2015. Occlusion: a contemporary overview and recommendations. Int. J. Periodontics Restorative Dentistry. 35: 775-782.

30. Robinson L.J., Durham J., Newton J.L. 2016. A systematic review of the comorbidity between temporomandibular disorders and chronic fatigue syndrome. J. Oral Rehabil. 43: 306-316.

31. Saito H., Katagiri A., Okada S., Mikuzuki L., Kubo A., Suzuki T., Ohara K., Lee J., Gionhaku N., Iinuma T., Bereiter D.A., Iwata K. 2017. Ascending projections of nociceptive neurons from trigeminal subnucleus caudalis: a population approach. Exp. Neurol. 293: 124-136.

32. Sessle B.J., Hu J.W., Amano N., Zhong G. 1986. Convergence of cutaneous, tooth pulp, visceral, neck and muscle afferents onto nociceptive and non nociceptive neurones in trigeminal subnucleus caudalis (medullary dorsal horn) and its implications for referred pain. Pain. 27: 219-235.

33. Shankland W.E. II. 2000. The trigeminal nerve. Part 1: an over-view. Cranio. 18: 238-248.

34. Shephard M.K., MacGregor E.A., Zakrzewska J.M. 2013. Orofacial pain: a guide for the headache physician. Headache. 54: 22-39.

35. Sheridan R.A., Decker A.M., Plonka A.B., Wang H.L. 2016. The role of occlusion in implant therapy: a comprehensive updated review. Implant Dent. 2: 829-838.

36. Tecco S., Marzo G., Crincoli V., Di Bisceglie B., Tete S., Festa F. 2012. The prognosis of myofascial pain syndrome (MPS) during a fixed orthodontic treatment. Cranio. 30 (1): 52-71.

37. Tkachenko I.M., Vodoriz Y.Y., Marchenko I.Y., Shundryk M.A., Korol D.M., Gurzhiy O.V. 2019. Changes in electromyography test results of patients with pathological abrasion of teeth: the role of anterior teeth in the process of rehabilitation. Wiad Lek. 72 (4): 553-557.

38. Vadivelu N., Mitra S., Schermer E., Kodumudi V., Kaye A.D., Urman R.D. 2014. Preventive analgesia for postoperative pain control: a broader concept. Local Reg Anesth. 7: 17-22.

39. Yau H.T., Yang T.J., Chen Y.C. 2014. Tooth model reconstruction based upon data fusion for orthodontic treatment simulation. Comput Biol Med. 48: 8-16.

40. Yu C.Y., Abbott P.V. 2016. Responses of the pulp, periradicular and soft tissues following trauma to the permanent teeth. Aust. Dent. J. 61 (1): 39-58.

\section{References}

1. Iordanishvili A.K., Serikov A.A. 2018. O neobkhodimosti standartizaczii podkhodov k lecheniyu i reabilitaczii paczientov s patologiej visochno-nizhnechelyustnogo sustava $\mathrm{v}$ uchrezhdeniyakh raznoj formy sobstvennosti [About the need to standardize approaches to the treatment and rehabilitation of patients with pathology of the temporomandibular joint in institutions of different ownership forms]. Mediczinskij vestnik Severnogo Kavkaza. 13 (1-1): 59-62.

2. Ordokova E.R. 2018. Diagnostika i lechenie disfunkczii visochno-nizhnechelyustnogo sustava u paczientov s anomaliyami prikusa [Diagnosis and treatment of temporomandibular joint dysfunction in patients with malocclusion]. Smolenskij mediczinskij almanakh. 2: 55-57.

3. Silaev A.M., Zubova K.N., Novoselczev S.V. 2014. Osteopaticheskie vozmozhnosti diagnostiki i lecheniya sindroma disfunkczii visochno-nizhnechelyustnogo sustava [Osteopathic opportunities for the diagnosis and treatment of temporomandibular joint dysfunction syndrome]. Manualnaya terapiya. 4: $20-31$.

4. Abduo J., Tennant M., McGeachie J. 2013. Lateral occlusion schemes in natural and minimally restored permanent dentition: a systematic review. J Oral Rehabil. 40: 788-802.

5. Al-Salleh M.A., Armijo-Olivo S., Flores-Mir C., Thie N.M. 2012. Electromyography in diagnosing temporomandibular disorders. J Am Dent Assoc. 143: 351-362.

6. Baad-Hansen L., Benoliel R. 2017. Neuropathic orofacial pain: facts and fifiction. Cephalalgia. 37: 670-679.

7. Bakke M., Petersson A., Wiesel M., Svanholt P., Sonnesen L. 2014. Bony dethroughtions revealed by cone beam computed tomography of the temporomandibular joint in subjects without ongoing pain. J Oral Facial Pain Headache. 28: 331-337.

8. $\quad$ Chung F., Subramanyam R., Liao P., Sasaki E., Shapiro C., Sun Y. 2012. High stop bang score indicates a high probability of obstructive sleep apnoea. Br J Anaesth. 108: 768-775. 
9. Dolce M.C., Aghazadeh-Sanai N., Mohammed S., Fulmer T.T. 2014. Integrating oral health into the interdisciplinary health sciences curriculum. Dent Clin North Am. 58: 829-843.

10. Durham J., Raphael K.G., Benoliel R., Ceusters W., Michelotti A., Ohrbach R. 2015. Perspectives on next steps in classifification of oro-facial pain - part 2: role of psychosocial factors. J Oral Rehabil. 42: 942-955.

11. Emodi Perelman A., Eli I., Rubin P.F., Greenbaum T., Heiliczer S., Winocur E. 2015. Occupation as a potential contributing factor for temporomandibular disorders, bruxism, and cervical muscle pain: a controlled comparative study. Eur J Oral Sci. 123 (5): 356-361.

12. Ferro K.J., Morgano S.M., Driscoll C.F., Freilich M.A., Guckes A.D., Knoernschild K.L., VcGarry T.J. 2017. The glossary of prosthodontic terms. Ninth edition. J. Prosthet. Dent. 117 (5): 1-105.

13. Fishbain D.A., Pulikal A., Lewis J.E., Gao J. 2017. Chronic pain types differ in their reported prevalence of post-traumatic stress disorder (PTSD) and there is consistent evidence that chronic pain is associated with ptsd: an evidence based structured systematic review. Pain Med. 18: 711-735.

14. Gauer R.L., Semidey M.J. 2015. Diagnosis and treatment of temporomandibular disorders. Am Fam Physician. 91: 378-386.

15. Ghurye S., McMillan R. 2017. Orofacial pain: an update on diagnosis and man agement. Br. Dent. J. 223: 639-647.

16. Greene C.S., Obrez A. 2015. Treating temporomandibular disorders with permanent mandibular repositioning: is it medically necessary? Oral Surg., Oral Med., Oral Pathol., Oral Radiol. 119: 489-498.

17. Hu J.L., Dong Y. 2019. Research progress in occlusal splint therapy for disorders. Zhonghua Kou Qiang Yi Xue Za Zhi. 54 (4): 273-277.

18. Kretschmer W.B., Baciuţ G., Baciuţ M., Sader R. 2019. Effect of bimaxillary orthognathic surgery on dysfunction temporomandibular joint: a retrospective study of 500 consecutive cases. Br. J. Oral Maxillofac. Surg. 57 (8): 734-739.

19. Manfredini D., Serra-Negra J., Carboncini F., Lobbezoo F. 2017. Current concepts of bruxism. Int. J. Prosthodont. 30: 437-438.

20. McSwiney T.P., Collins J.M., Bassi G.S., Khan S. 2017. The interdisciplinary management of hypodontia patients in the UK: a national service evaluation. Br. Dent. J. 222 (1): 31-35.

21. Melzack R. 2001. Pain and the neuromatrix in the brain. J. Dent. Educ. 65: 1378-1382.

22. Merrill R.L., Goodman D. 2016. Chronic orofacial pain and behavioral medicine. Oral Maxillofac. Surg. Clin. North. Am. 28: 247-260.

23. Milosevic A. 2017. Abrasion: A Common Dental Problem Revisited. Prim. Dent. J. 6 (1): $32-36$.

24. Moreno-Hay I., Okeson J.P. 2015. Does altering the occlusal vertical dimension produce temporomandibular disorders? A literature review. J. Oral Rehabil. 42: 875-882.

25. Mousa M.A., Patil S., Lynch E. 2017. Masticatory effificiency and muscular activity in removable partial dental prostheses with different cusp angles. J. Prosthet. Dent. 117: 55-60.

26. Ohkubo C., Morokuma M., Yoneyama Y., Matsuda R., Lee J.S. 2013. Interactions between occlusion and human brain function activities. J. Oral Rehabil. 40: 119-129.

27. Ohrbach R., Dworkin S.F. 2016. The evolution of TMD diagnosis: past, present, future. J. Dent. Res. 95: 1093-1101.

28. Porwal A., Sasaki K. 2013. Current status of the neutral zone: a literature review. J. Prosthet. Dent. 109: 129-134.

29. Racich M.J. 2015. Occlusion: a contemporary overview and recommendations. Int. J. Periodontics Restorative Dentistry. 35: 775-782.

30. Robinson L.J., Durham J., Newton J.L. 2016. A systematic review of the comorbidity between temporomandibular disorders and chronic fatigue syndrome. J. Oral Rehabil. 43: 306-316.

31. Saito H., Katagiri A., Okada S., Mikuzuki L., Kubo A., Suzuki T., Ohara K., Lee J., Gionhaku N., Iinuma T., Bereiter D.A., Iwata K. 2017. Ascending projections of nociceptive neurons from trigeminal subnucleus caudalis: a population approach. Exp. Neurol. 293: 124-136.

32. Sessle B.J., Hu J.W., Amano N., Zhong G. 1986. Convergence of cutaneous, tooth pulp, visceral, neck and muscle afferents onto nociceptive and non nociceptive neurones in trigeminal subnucleus caudalis (medullary dorsal horn) and its implications for referred pain. Pain. 27: 219-235.

33. Shankland W.E. II. 2000. The trigeminal nerve. Part 1: an over-view. Cranio. 18: 238-248. 
34. Shephard M.K., MacGregor E.A., Zakrzewska J.M. 2013. Orofacial pain: a guide for the headache physician. Headache. 54: 22-39.

35. Sheridan R.A., Decker A.M., Plonka A.B., Wang H.L. 2016. The role of occlusion in implant therapy: a comprehensive updated review. Implant Dent. 2: 829-838.

36. Tecco S., Marzo G., Crincoli V., Di Bisceglie B., Tete S., Festa F. 2012. The prognosis of myofascial pain syndrome (MPS) during a fixed orthodontic treatment. Cranio. 30 (1): 52-71.

37. Tkachenko I.M., Vodoriz Y.Y., Marchenko I.Y., Shundryk M.A., Korol D.M., Gurzhiy O.V. 2019. Changes in electromyography test results of patients with pathological abrasion of teeth: the role of anterior teeth in the process of rehabilitation. Wiad Lek. 72 (4): 553-557.

38. Vadivelu N., Mitra S., Schermer E., Kodumudi V., Kaye A.D., Urman R.D. 2014. Preventive analgesia for postoperative pain control: a broader concept. Local Reg Anesth. 7: 17-22.

39. Yau H.T., Yang T.J., Chen Y.C. 2014. Tooth model reconstruction based upon data fusion for orthodontic treatment simulation. Comput Biol Med. 48: 8-16.

40. Yu C.Y., Abbott P.V. 2016. Responses of the pulp, periradicular and soft tissues following trauma to the permanent teeth. Aust. Dent. J. 61 (1): 39-58.

\section{Ссылка для цитирования статьи For citation}

Дзалаева Ф.К., Чикунов С.О., Утюж А.С., Михайлова М.В., Будунова М.К. 2020. Междисциплинарный подход в лечении орофациальной боли и патологии височнонижнечелюстного сустава у пациентов с полным или частичным отсутствием зубов (обзор литературы). Актуальные проблемы медицины, 43(1): 102-112. DOI

Dzalaeva F.K., Chikunov S.O., Utyuzh A.S., Mikhailova M.V., Budunova M.K. 2020. An interdisciplinary approach in the treatment of orofacial pain and pathology of the temporomandibular joint in patients with complete or partial adentia (review of literature). Challenges in Modern Medicine, 43(1): 102-112 (in Russian). DOI 